\title{
Is hemodynamic transesophageal echocardiography needed for patients with left ventricular assist device?
}

\author{
Nataliya Bahatyrevich, BA, Qiong Yang, MD, Nicholas C. Cavarocchi, MD, and Hitoshi Hirose, MD
}

\section{ABSTRACT}

Background: Interventions in patients with a left ventricular assist device (LVAD) in the intensive care unit (ICU) are typically performed based on the results of conventional monitoring, such as vital signs and Swan-Ganz catheter (SGC) and LVAD parameters. These variables might not always accurately reflect a patient's cardiac function, volume status, and interventricular septal configuration, however. To assess the accuracy of standard monitoring, we performed routine continuous hemodynamic transesophageal echocardiography (hTEE) to evaluate cardiac function, volume status, and septal position.

Methods: Between 2011 and 2015, 93 HeartMate II LVADs were implanted. The study group comprised 30 patients with an SGC in place who were monitored routinely by hTEE in the ICU every 1 to 3 hours until extubation. A total of 147 hTEE studies were analyzed retrospectively to observe differences between conventional monitoring and hTEE.

Results: Among the 30 patients studied, $26(87 \%)$ had at least 1 disagreement between conventional monitoring and hTEE findings. In 22 patients $(73 \%)$, at least 1 of the hTEE studies was abnormal whereas conventional parameters were normal. Abnormal hTEE findings included a shift in the interventricular septum in 19 patients (63\%), abnormal ventricular volume status in 22 patients $(73 \%)$, and right ventricular failure in 9 patients $(30 \%)$. Based on conventional monitoring, none of the patients required an LVAD speed change, whereas hTEE showed that 14 patients $(47 \%)$ needed an LVAD speed adjustment.

Conclusions: Conventional monitoring in the ICU might not provide an accurate representation of cardiac function, ventricular volume status, or septal position in patients with LVAD. Continuous monitoring with hTEE in patients with an LVAD may help guide optimal intervention in the ICU setting during the early postoperative period. (J Thorac Cardiovasc Surg 2018;155:1071-7)

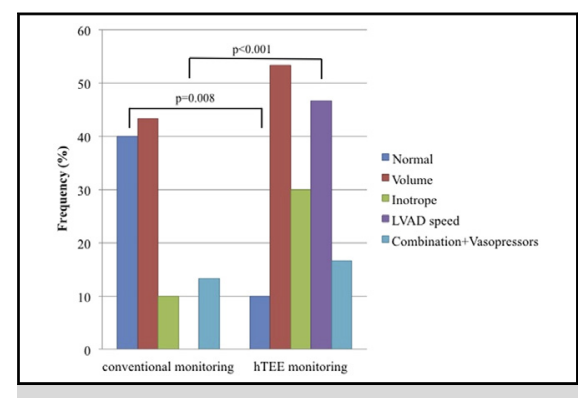

Comparison of interventions in 30 patients from conventional hemodynamic monitoring and hTEE monitoring. hTEE monitoring often suggests LVAD speed change.

\section{Central Message}

Swan-Ganz monitoring might not be sufficient to monitor cardiac function and volume status of patients with LVAD. We advocate the use of hTEE as an adjunct for patient monitoring.

\section{Perspective}

Interventions in patients with LVAD are typically based on conventional monitoring, which includes Swan-Ganz catheter parameters. We found that conventional monitoring might not always accurately reflect a patient's cardiac function, volume status, and interventricular septal position. Additional hTEE monitoring may be beneficial in choosing the optimal intervention for patients with LVAD.

See Editorial Commentary page 1078.

See Editorial page 1058
Postoperative management in the intensive care unit (ICU) of patients who underwent left ventricular assist device (LVAD) placement is typically based on clinical variables, such as Swan-Ganz catheter (SGC) parameters, vital signs, and LVAD device parameters. An SGC is placed

\footnotetext{
From the Division of Cardiothoracic Surgery, Department of Surgery, Thomas Jefferson University, Philadelphia, Pa.

Received for publication Nov 1, 2016; revisions received Aug 30, 2017; accepted for publication Sept 28, 2017; available ahead of print Dec 14, 2017.

Address for reprints: Hitoshi Hirose, MD, Department of Surgery, Thomas Jefferson

University, 1025 Walnut St, Room 605, Philadelphia, PA 19107 (E-mail: Hitoshi.

Hirose@jefferson.edu).

$0022-5223 / \$ 36.00$

Copyright (c) 2017 by The American Association for Thoracic Surgery

https://doi.org/10.1016/j.jtcvs.2017.09.142
}

intraoperatively to monitor pulmonary pressure, cardiac output, and vascular resistance. The benefit of an SGC in patients who have undergone cardiac surgery has been widely debated. ${ }^{1}$ Several studies have shown that SGC use may provide little benefit or lead to worse outcomes in septic, critically ill, or high-risk surgical patients. ${ }^{2-5}$ How accurately SGC parameters reflect cardiac function,

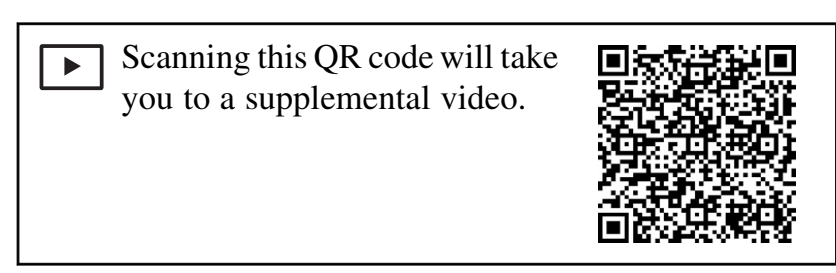




\section{Abbreviations and Acronyms \\ $\mathrm{CVP}=$ central venous pressure \\ $\mathrm{ECMO}=$ extracorporeal membrane oxygenation \\ hTEE = hemodynamic transesophageal echocardiography \\ ICU = intensive care unit \\ LVAD $=$ left ventricular assist device \\ $\mathrm{MAP}=$ mean arterial pressure \\ SGC = Swan-Ganz catheter}

interventricular septal position, or ventricular volume status in patients with an LVAD remains unclear.

As reported previously, ${ }^{6}$ hemodynamic transesophageal echocardiography (hTEE) is a disposable, flexible 5.5-mmdiameter transesophageal probe that has been approved by the Food and Drug Administration for cardiac monitoring of patients in the ICU for 72 hours per probe. The probe allows physicians to perform rapid qualitative and semiquantitative assessment of cardiac function, ventricular volume status, and ventricular septal configuration. ${ }^{7}$ In particular, hTEE findings can be used to adjust LVAD speed based on interventricular septal position and help guide proper fluid and inotrope administration, relying on the observed right ventricular function and ventricular volume status. Information obtained from echocardiography has been used for postoperative management of cardiac surgery patients, ${ }^{8}$ weaning off of extracorporeal membrane oxygenation (ECMO), ${ }^{9}$ and diagnosis of postcardiotomy tamponade. $^{10}$

A recent retrospective review showed that postoperative hTEE is typically performed in sicker patients who are greater risk of morbidity and mortality following LVAD implantation. ${ }^{7}$ Furthermore, the study reported changes in clinical management based on hTEE findings in the majority of these patients. ${ }^{7}$ We hypothesized that conventional monitoring with an SGC in patients with an LVAD might not reflect ventricular volume status, right ventricular function, and interventricular septal configuration as accurately has been demonstrated by point-of-care hTEE monitoring, and that routine hTEE could lead to changes in the clinical management of patients with LVAD, even in those with standard SGC monitoring.

\section{METHODS}

Between May 2011 and May 2015, a total of 93 HeartMate II LVADs (Thoratec, Pleasanton, Calif) were implanted in our hospital. All 93 patients with an LVAD underwent conventional hemodynamic monitoring with an SGC. Among the 93 patients, 30 patients also underwent routine continuous hTEE (ImaCor hTEE; ImaCor, Garden City, NY), performed every 1 to 3 hours by qualified intensivists, in addition to conventional SGC monitoring and recording of LVAD parameters. The cost of the hTEE was approximately $\$ 1250$ per probe. Eligibility criteria for the hTEE study included the presence of hTEE-trained personnel and availability of the hTEE device. A total of 147 hTEE studies were conducted in 30 patients until extubation under standard sedation used routinely after cardiac surgery. The hTEE images were retrospectively retrieved from the console after approval from the Thomas Jefferson University's Institutional Review Board (IRB \#11D.451). All hTEE studies yielded images of adequate quality for review and analysis. The data from conventional hemodynamic monitoring, including LVAD and SGC parameters, patient demographic information, and hTEE recordings, were stored in a database for our retrospective analysis.

Conventional hemodynamic monitoring included mean arterial pressure (MAP), heart rate, central venous pressure (CVP), pulmonary artery systolic and diastolic pressure, cardiac index, and LVAD parameters, which included speed, flow, pulsatility index, and power. Based on conventional monitoring, volume status was defined based on low CVP, MAP, and LVAD pulsatility index. Based on hTEE, volume status was evaluated and defined as euvolemia, hypovolemia, or hypervolemia, based on left ventricular cavity size. The position of the interventricular septum was evaluated by hTEE and categorized as midline, shifted to the right, or shifted to the left. By conventional monitoring, right ventricular failure was defined as high CVP with a concomitant low cardiac index. By hTEE, right ventricular function was categorized as hypokinetic, normal, or hyperdynamic. No patient had right ventricular failure necessitating mechanical support. The management algorithm for conventional monitoring and a potential patient management algorithm for monitoring by hTEE are shown in Figure 1.

Data for conventional hemodynamic monitoring (without hTEE) were analyzed using absolute numbers and trends in parameters from previous hTEE study time points. Based solely on these conventional parameters, patients were divided into 2 groups, a group requiring intervention(s), such as adjustments of volume, vasopressors, and inotropes and changes in LVAD speed, and a normal group, defined as not requiring intervention, at each time point. A similar analysis was performed taking hTEE findings into consideration. The interventions suggested by conventional monitoring results were compared with those suggested by hTEE findings.

Demographic data, preimplantation diagnoses, postoperative SGC parameters and LVAD settings, and reasons for LVAD implantation were also compared between the 63 patients who did not receive hTEE monitoring (non-hTEE group) and the 30 patients who received hTEE monitoring (hTEE group). Survival at 30 days, 6 months, 12 months, and 24 months was analyzed using a Kaplan-Meier curve.

Data are expressed as number with percentage, mean \pm standard deviation with range, or mean \pm standard deviation and median with range and interquartile range. Statistical comparisons between the conventional monitoring and hTEE groups were performed using the $\chi^{2}$ or Fisher exact test, as appropriate, for categorical variables, and using the standard $t$ test or Mann-Whitney $U$ test, as appropriate, for continuous variables. Cohen's $\kappa$, reflecting the agreement between conventional monitoring and hTEE, was calculated. Log-rank test $P$ values and $95 \%$ confidence intervals were calculated for Kaplan-Meyer analysis. A $P$ value $<.05$ was considered to indicate significance.

\section{RESULTS}

Routine postoperative hTEE studies were performed in 30 patients who underwent LVAD implantation and compared with SGC and LVAD data. A mean of $5 \pm 2$ hTEE studies (range, 3-12) per patient were performed during the postoperative period. There were no adverse events related to hTEE, such as esophageal injury or oropharyngeal injury, during the study period. The 30 patients in the hTEE group included 23 males and 7 females, with a mean age of $56 \pm 12$ years (Table 1 ). The 30-day survival was $100 \%$ in this group, but 6 patients 

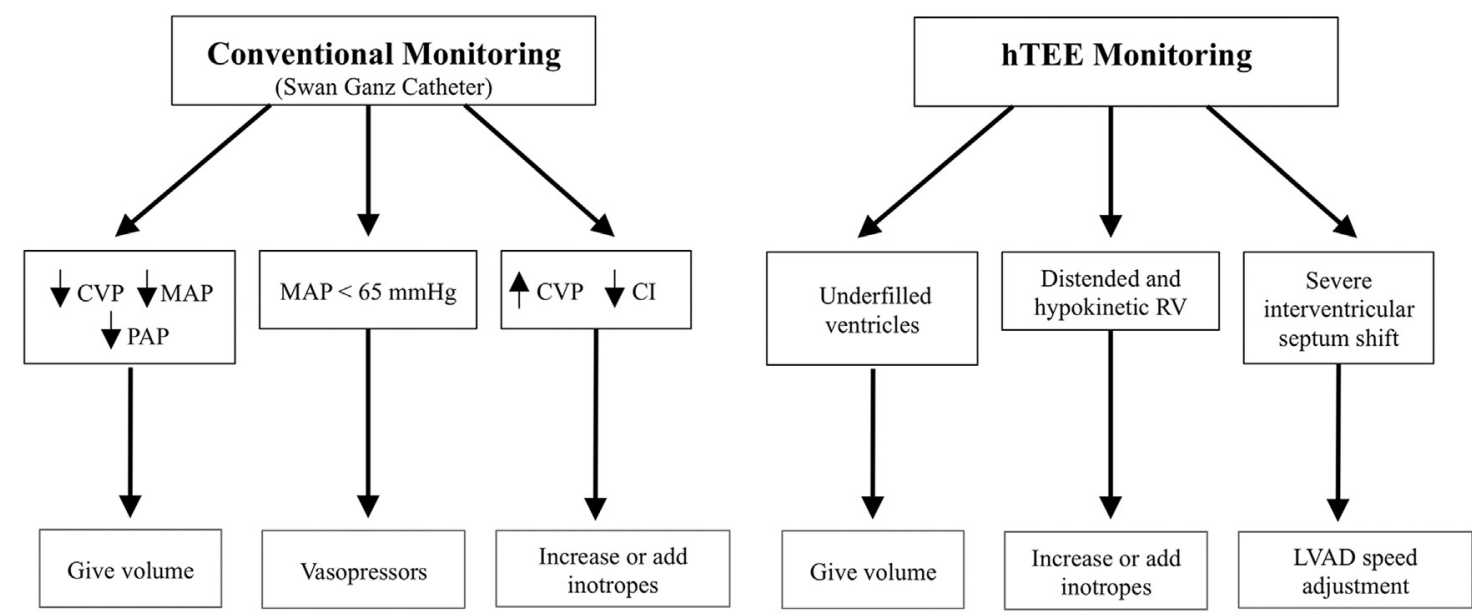

FIGURE 1. Flow chart of the algorithm for each intervention in conventional monitoring and hTEE monitoring. hTEE, Hemodynamic transesophageal echocardiography; $C V P$, central venous pressure; $M A P$, mean arterial pressure; $P A P$, pulmonary artery pressure; $C I$, cardiac index; $R V$, right ventricle; $L V A D$, left ventricular assist device.

$(20 \%)$ died after 30 days (Table 1). A comparison of demographic data between the 63 patients in the non-hTEE group and the 30 patients in the hTEE group showed no statistical differences in sex, age, length of ICU and hospital stay, or LVAD end therapy (Table 1). Comparison of 30-day survival between the non-hTEE and hTEE groups showed a statistically significant difference $(87 \%$ vs $100 \% ; P=.040)$ (Table 1$)$. Preimplantation diagnoses and reasons for LVAD implantation are compared between the 2 groups in Table 2. Statistically significant differences in preimplantation diagnoses were observed only in the transition from ECMO to LVAD, with a higher percentage of post-ECMO patients in the non-hTEE group ( $13 \%$ vs $0 \%$ in the hTEE group; $P=.040)$. A comparison of conventional parameters in the immediate postoperative period on transfer to the ICU between the 2 groups found statistically significant differences in MAP, pulmonary systolic pressure, and LVAD parameters (Table 3). Specifically, MAP, pulmonary systolic pressure, and pulsatility index were lower in the non-hTEE group. Kaplan-Meyer analysis comparing survival in the 2 groups at 1 month, 6 months, 12 months, and 24 months is shown in Figure 2. Survival at 24 months was lower in the non-hTEE group $(63.5 \%$ vs $80 \%)$.

Hemodynamic parameters in the hTEE group are presented in Table 4. Interpretation of the hTEE and implied interventions based on hTEE findings are shown in Tables 5 and 6 , respectively. The proportion of agreement between conventional monitoring and hTEE monitoring for each intervention based on 147 studies is shown in Table 6, with the lowest agreement seen between LVAD speed change and inotropes ( 0 and 0.14 , respectively). The conventional hemodynamic monitoring results from the SGC and results from hTEE disagreed in 70 of the 147 studies $(48 \%)$. The simple unweighted $\kappa$ coefficient between conventional and hTEE-monitored groups was 0.22 , suggesting disagreement between the SGC and hTEE findings. Among the 30 patients in the hTEE group, $26(87 \%)$ had at least 1 disagreement between SGC and hTEE findings throughout the entire hTEE study period. In 22 patients $(73 \%)$, at least 1 hTEE study showed that routine

TABLE 1. Comparison of patient demographics in the non-hTEE and hTEE groups

\begin{tabular}{|c|c|c|c|}
\hline Demographics & Non-hTEE $(n=63)$ & hTEE $(n=30)$ & $P$ value \\
\hline Male sex, $\mathrm{n}(\%)$ & $51(81)$ & $23(77)$ & .6556 \\
\hline Age, $y$, mean $\pm \mathrm{SD}$; median (range, IQR) & $58 \pm 11 ; 61(27-74,14.5)$ & $56 \pm 12,59(22-75,17.5)$ & .4179 \\
\hline $\mathrm{ICU}$ stay, $\mathrm{d}$, mean $\pm \mathrm{SD}$; median (range, IQR) & $8.9 \pm 9.1 ; 5.5(3-51,4.8)$ & $12.0 \pm 17.3,6.5(4-84,4.0)$ & .1556 \\
\hline Hospital stay, d, mean $\pm \mathrm{SD}$; median (range, IQR) & $20.8 \pm 12.5 ; 15.5(4-59,9.8)$ & $23.4 \pm 17.0,19(8-93,12.8)$ & .5823 \\
\hline 30-d survival, n (\%) & $55(87)^{*}$ & $30(100)$ & .0397 \\
\hline \multicolumn{4}{|l|}{ End therapy, n (\%) } \\
\hline Bridge to transplantation/decision & $28(44)$ & $15(50)$ & .5893 \\
\hline Destination & $35(56)$ & $15(50)$ & .5893 \\
\hline
\end{tabular}

hTEE, Hemodynamic transesophageal echocardiography; $S D$, standard deivation; $I Q R$, interquartile range; $I C U$, intensive care unit. *One patient died in the operating room. 
TABLE 2. Comparison of diagnoses and reasons for LVAD implantation between non-hTEE and hTEE groups

\begin{tabular}{|c|c|c|c|}
\hline Reasons & $\begin{array}{l}\text { Non-hTEE } \\
(\mathbf{n}=\mathbf{6 3}) \\
\text { n }(\%)\end{array}$ & $\begin{array}{c}\text { hTEE } \\
(\mathrm{n}=\mathbf{3 0}), \\
\text { n }(\%)\end{array}$ & $\begin{array}{c}P \\
\text { value }\end{array}$ \\
\hline Inotrope-dependent & $33(52)$ & $20(67)$ & .1745 \\
\hline Class IV CHF & $58(92)$ & $28(93)$ & .8663 \\
\hline Hypertrophic cardiomyopathy & $1(2)$ & $1(3)$ & .7659 \\
\hline Ischemic cardiomyopathy & $18(29)$ & $6(20)$ & .3582 \\
\hline Non-Ischemic cardiomyopathy & $40(63)$ & $21(70)$ & .5098 \\
\hline LVAD malfunction/thrombus & $5(8)$ & $3(10)$ & .7497 \\
\hline Weaning off ECMO & $8(13)$ & $0(0)$ & .0397 \\
\hline
\end{tabular}

parameters were diagnosed as normal while the hTEE diagnosis was abnormal.

Among the 147 hTEE studies, conventional hemodynamic assessments were normal in 79\% (116 of 147), whereas hTEE demonstrated normal volume and functional status in only $44 \%$ ( 65 of $147 ; P<.001)$. The suggested interventions based on abnormal SGC or hTEE findings were as follows: need for volume adjustment, $16 \%$ (24 of 147) by SGC assessment before hTEE versus $27 \%$ (39 of 147) by hTEE $(P=.03)$, need for inotrope adjustment due to right ventricular failure, 2\% (3 of 147) by SGC assessment before hTEE versus $9 \%$ (13 of 147$)$ by hTEE $(P=.01)$; need for LVAD speed change, $0 \%$ ( 0 of 147) by SGC assessment before hTEE versus $17 \%$ ( 25 of 147$)$ by hTEE $(P<.001)$; need for a combination of interventions or vasopressors, $3 \%$ (4 of 147) by SGC assessment before hTEE versus 3\% (5 of 147) by hTEE $(P=.73)$ (Figure 3 ).

Among the 30 patients in the hTEE group, when comparing the proposed intervention by conventional hemodynamic assessments and hTEE, conventional monitoring was normal in $12(40 \%)$, whereas hTEE was normal in only $3(10 \%)(P=.008)$. Conventional hemodynamic assessment did not demonstrate the need for a speed change ( 0 patients; $0 \%)$; however, hTEE findings did show the need for a speed change (14 patients; $47 \%)(P<.001)$. The numbers of patients who were in need of a volume adjustment $(P=.44)$, inotropes $(P=.05)$, or a combination of interventions $(P=.71)$ did not differ significantly between the 2 groups (Figure 4 ).

The shift in the septum was frequently observed by hTEE (Video 1). Nineteen patients $(63 \%)$ had an interventricular septal configuration not in the midline. The septum was shifted to the right in 12 patients $(40 \%)$, to the left in 4 patients $(13 \%)$, and undetermined in 3 patients $(10 \%)$ (Figure 5), which means that in one hTEE study the septum was shifted to the right and in another hTEE study the septum was shifted to the left. In 58 hTEE studies (39\%), the ventricular volume was abnormal in either one or both ventricles. The majority of volume abnormalities were a hypovolemic right ventricle, observed in 20 studies $(14 \%)$, and hypervolemia in the left ventricle, observed in 15 studies $(10 \%)$. Overall, 22 patients $(73 \%)$ had an abnormal ventricular volume status (Table 5). Out of 147 studies, 3 showed right ventricular failure based on conventional monitoring in 3 different patients, which was addressed with inotropes. Hemodynamic TEE identified an additional 6 patients with right ventricular failure, whose conventional parameters were either normal ( 3 patients) or suggestive of hypovolemia ( 3 patients).

In addition, only 2 patients $(7 \%)$ received all of the interventions based on hTEE findings throughout the entire hTEE monitoring period. Out of 147 hTEE studies, 70 $(48 \%)$ led to the intervention recommended by the hTEE findings, $67(46 \%)$ did not lead to the recommended intervention, and $10(7 \%)$ led to additional interventions beyond that recommended based on the study findings.

TABLE 3. Comparison of conventional parameters between the non-hTEE and hTEE groups with calculated $P$ values

\begin{tabular}{|c|c|c|c|}
\hline Parameter & Non-hTEE $(n=63)$, mean \pm SD $($ range $)$ & hTEE $(n=30)$, mean \pm SD $($ range $)$ & $P$ value \\
\hline Mean arterial pressure, $\mathrm{mm} \mathrm{Hg}$ & $78 \pm 8(60-96)$ & $86 \pm 12(66-112)$ & .0003 \\
\hline Heart rate, bpm & $93 \pm 17(66-139)$ & $89 \pm 16(61-135)$ & .2827 \\
\hline Pulmonary systolic pressure, $\mathrm{mm} \mathrm{Hg}$ & $37 \pm 9(21-57)$ & $41 \pm 9(25-59)$ & .0481 \\
\hline Pulmonary diastolic pressure, $\mathrm{mm} \mathrm{Hg}$ & $20 \pm 6(10-37)$ & $21 \pm 5(13-32)$ & .4311 \\
\hline Central venous pressure, $\mathrm{mm} \mathrm{Hg}$ & $12 \pm 4(3-22)$ & $13 \pm 4(5-22)$ & .2627 \\
\hline Cardiac index, $\mathrm{L} / \mathrm{min} / \mathrm{m}^{2}$ & $2.6 \pm 0.5(1.4-4)$ & $2.4 \pm 0.5(1.4-3.6)$ & .0747 \\
\hline Mixed venous oxygen saturation, $\%$ & $66 \pm 8(43-83)$ & $63 \pm 8(49-80)$ & .0944 \\
\hline \multicolumn{4}{|l|}{ LVAD settings } \\
\hline Speed, rpm & $9070 \pm 330(8400-10,200)$ & $8900 \pm 310(8400-9600)$ & .0200 \\
\hline Flow, L/min & $4.7 \pm 1.1(2.6-8.8)$ & $4.2 \pm 1.0(2.4-7.3)$ & .0378 \\
\hline Pulsatility index & $4.6 \pm 1.4(2.1-8.3)$ & $6.2 \pm 1.7(3.3-9.0)$ & .0001 \\
\hline Power, W & $5.6 \pm 1.0(4.1-10)$ & $4.8 \pm 0.7(3.8-7)$ & .0002 \\
\hline
\end{tabular}

hTEE, Hemodynamic transesophageal echocardiography; $S D$, standard deviation; $L V A D$, left ventricular assist device. 


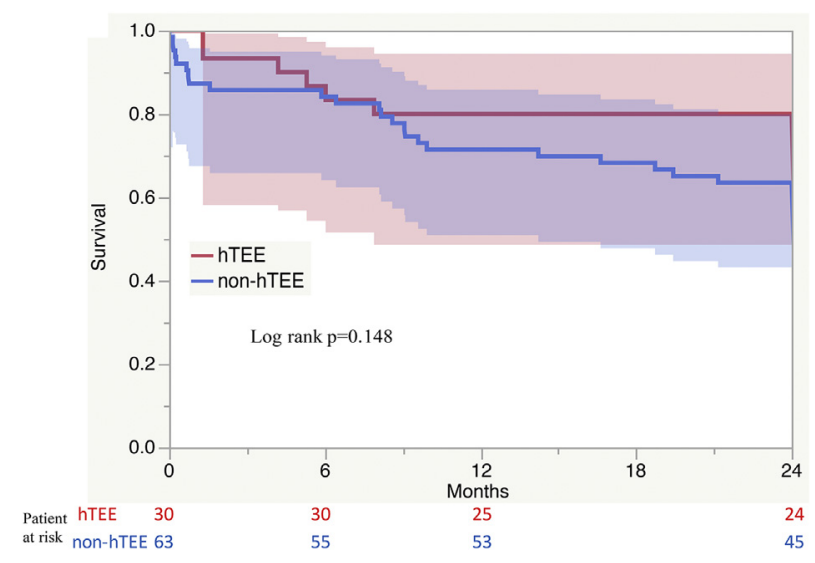

FIGURE 2. Kaplan-Maier plot comparing survival between non-hTEE (blue solid line) and hTEE (red solid line) groups at 1 month, 6 months, 12 months, and 24 months since the date of the procedure. The number of patients at risk is indicated for the 1-, 6-, 12-, and 24-month points below the graph (red for hTEE; blue for non-hTEE). The shaded area represents the $95 \%$ confidence interval for each group (red for hTEE; blue for non-hTEE). $P$ values (log-rank test) are shown. hTEE, Hemodynamic transesophageal echocardiography.

\section{DISCUSSION}

The interventions during the early postoperative period in the ICU following LVAD implantation typically include volume adjustment, inotrope adjustment, vasopressors, and LVAD speed changes. A flow chart summarizing the different algorithms for choosing a particular intervention based on conventional monitoring or on hTEE is shown in Figure 1. In cases of hypovolemia, the patient receives either colloid infusion (typically $250-500 \mathrm{~mL}$ of $5 \%$ albumin), or blood transfusion if hemoglobin is $<9 \mathrm{~g} / \mathrm{dL} .{ }^{11}$ Echocardiography in hypovolemia shows underfilled ventricles. ${ }^{11}$ In cases of right-sided heart failure, conventional monitoring typically shows a high CVP and a

TABLE 4. Swan-Ganz parameters and LVAD settings at the time of the hTEE study $(n=147)$

\begin{tabular}{lc}
\hline \multicolumn{1}{c}{ Parameter } & Value, mean \pm SD (range) \\
\hline Mean arterial pressure, $\mathrm{mm} \mathrm{Hg}$ & $82 \pm 10(50-112)$ \\
Heart rate, bpm & $89 \pm 12(60-130)$ \\
\hline Pulmonary systolic pressure, $\mathrm{mm} \mathrm{Hg}$ & $38 \pm 9(20-60)$ \\
Pulmonary diastolic pressure, mm Hg & $20 \pm 5(9-35)$ \\
Central venous pressure, mm Hg & $14 \pm 5(4-26)$ \\
Cardiac index, L/min/m ${ }^{2}$ & $2.5 \pm 0.5(1.4-4.0)$ \\
Mixed venous oxygen saturation, \% & $65 \pm 10(41-87)$ \\
LVAD settings & \\
Speed, rpm & $8990 \pm 340(8000-9600)$ \\
Flow, L/min & $4.3 \pm 0.8(2.4-7.3)$ \\
Pulsatility index & $5.6 \pm 1.6(2.4-9.6)$ \\
Power, W & $4.9 \pm 0.7(3.1-7.6)$ \\
\hline
\end{tabular}

$S D$, Standard deviation; $L V A D$, left ventricular assist device.
TABLE 5. Summary of findings in hTEE studies and patients

\begin{tabular}{|c|c|c|}
\hline hTEE findings & $\begin{array}{c}\text { Studies } \\
(\mathrm{n}=147), \\
\text { n }(\%)\end{array}$ & $\begin{array}{c}\text { Patients } \\
(\mathbf{n}=\mathbf{3 0}), \\
\text { n }(\%)\end{array}$ \\
\hline Abnormal ventricular volume & $58(39)$ & $22(73)$ \\
\hline Right ventricular failure & $13(9)$ & $9(30)$ \\
\hline Interventricular septal shift & $32(22)$ & $19(63)$ \\
\hline Normal & $65(44)$ & $3(10)$ \\
\hline
\end{tabular}

hTEE studies may show multiple findings throughout the study for each patient Some patients may have different findings on hTEE during the postoperative period. hTEE, Hemodynamic transesophageal echocardiography.

low cardiac index, and echocardiography shows right atrium dilation and/or hypokinetic right ventricle contractility, frequently necessitating an increase in postoperative inotrope dosage following surgery or the addition of a new inotrope. ${ }^{11}$ Vasopressors are typically increased or added in hypotension (defined as an MAP $<65 \mathrm{~mm} \mathrm{Hg}$ ), although the use of vasopressors is determined not by hTEE, but more likely by conventional monitoring. ${ }^{11}$ Finally, LVAD speed, the sole parameter that can be adjusted with the device, plays an important role in mechanical unloading of the left ventricle, which affects many parameters of the cardiac status, including the function of the right ventricle. ${ }^{11}$ The optimal LVAD speed is obtained when left ventricular size and cardiac index are within normal ranges and the interventricular septum is midline. ${ }^{11}$ Unlike SGC monitoring, hTEE provides visualization of the septal position and thus allows for more optimal LVAD speed adjustment in the early postoperative period. The clinical utility of echocardiography for estimating right ventricular function, cardiac volume status, and interventricular septal position for appropriate intervention has been reported previously. ${ }^{12}$ In more than one-half of the patients $(52 \%)$, hTEE-measured hemodynamic values differed from conventional monitoring values. The majority of patients $(87 \%)$ had discrepant conventional hemodynamic monitoring and

TABLE 6. Suggested interventions based on hTEE findings and calculated proportion of agreement between conventional and hTEE monitoring for each intervention based on studies

\begin{tabular}{lccc}
\hline \multicolumn{1}{c}{ Interventions } & $\begin{array}{c}\text { Studies } \\
(\mathbf{n = 1 4 7 ) ,} \\
\mathbf{n}(\%)\end{array}$ & $\begin{array}{c}\text { Patients } \\
(\mathbf{n = 3 0 )} \\
\mathbf{n}(\%)\end{array}$ & $\begin{array}{c}\text { Proportion of } \\
\text { agreement } \\
(\mathbf{n}=\mathbf{1 4 7})\end{array}$ \\
\hline No need for intervention & $65(44)$ & $3(10)$ & 0.4959 \\
Volume & $39(27)$ & $16(53)$ & 0.2353 \\
Inotropes & $13(9)$ & $9(30)$ & 0.1429 \\
LVAD speed change & $25(17)$ & $14(47)$ & 0 \\
$\begin{array}{l}\text { Combination of above } \\
\text { or vasopressors }\end{array}$ & $5(3)$ & $5(17)$ & 0.5 \\
\hline $\begin{array}{l}\text { Individual patients may need several different interventions based on hTEE findings } \\
\text { during the postoperative period. } \text { LVAD, Left ventricular assist device. }\end{array}$
\end{tabular}




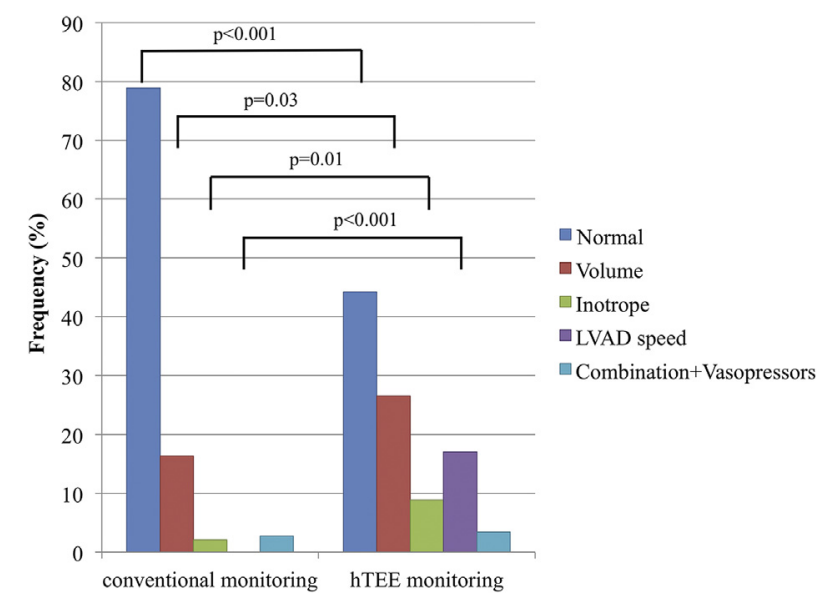

FIGURE 3. Comparison of requirements for various interventions in 147 studies based on conventional hemodynamic monitoring (conventional monitoring) and hTEE (hTEE monitoring) showing significant differences across the normal, volume, inotrope, and LVAD speed change groups. Combination represents the need for multiple interventions (both volume and speed change). LVAD speed represents the need for LVAD speed change. $L V A D$, Left ventricular assist device; hTEE, hemodynamic transesophageal echocardiography.

hTEE findings. Significant differences in suggested interventions for volume, inotropes, and LVAD speed change were observed when comparing $147 \mathrm{hTEE}$ studies and significant differences in LVAD speed change were seen when comparing 30 patients were observed out of hTEE study.

A comparison of the non-hTEE and hTEE groups showed improved overall survival and statistically significantly improved 30-day survival in the hTEE group. Major factors complicating this finding are significant between-group differences in conventional monitoring parameters and

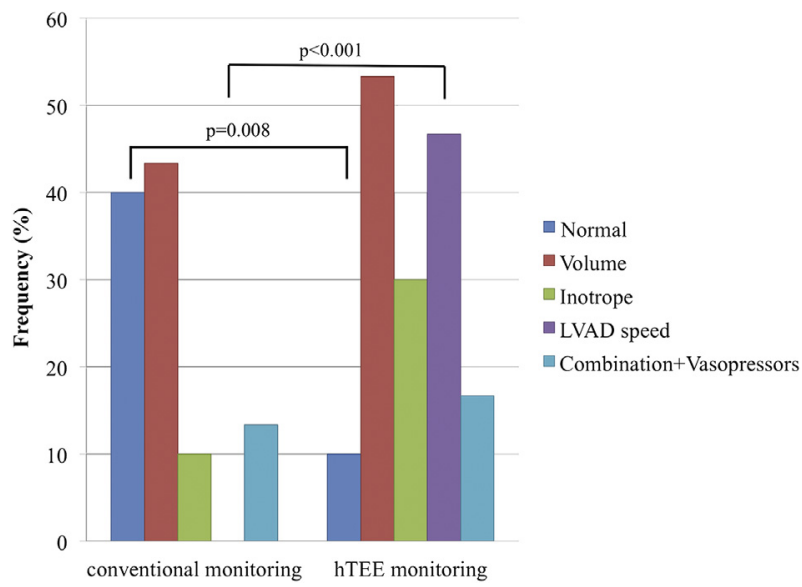

FIGURE 4. Comparison of interventions in 30 patients from conventional hemodynamic monitoring (conventional monitoring) and hTEE (hTEE monitoring) showing a significant difference between frequency of normal and LVAD speed change. Combination represents the need for multiple interventions (both volume and speed change). LVAD speed represents the need for LVAD speed change. LVAD, Left ventricular assist device; hTEE, hemodynamic transesophageal echocardiography.

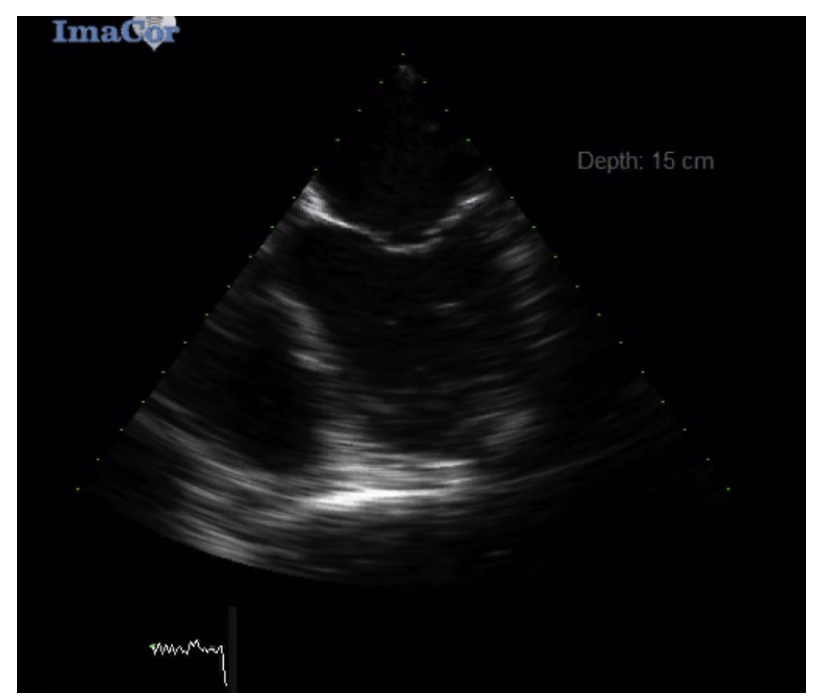

VIDEO 1. Example of hTEE finding showing bowing of interventricular septum toward the left ventricle, requiring decrease in speed on the LVAD settings in a patient with Swan-Ganz catheter parameters that do not require intervention (pulmonary pressure, $54 / 28 \mathrm{~mm} \mathrm{Hg}$; central venous pressure, $19 \mathrm{~mm} \mathrm{Hg}$; cardiac index, 2.7; mixed venous oxygen saturation, 58\%; LVAD speed, $8780 \mathrm{rpm}$; flow, $4.6 \mathrm{~L} / \mathrm{min}$; pulsatility index, 7.0, power, 5.0 W). Video available at: http://www.jtcvsonline.org/article/S00225223(17)32430-3/fulltext.

preimplantation diagnoses. Another limitation is the fact that only a small proportion of the patients in the hTEE group $(7 \%)$ received the interventions at the time of hTEE, because this study was not designed to perform all interventions based on hTEE findings. Therefore, in the hTEE group, all of the effects of hTEE on survival might not have been fully assessed. It is possible that other factors that we were not able to identify in this study might have played a role in the survival difference. Nevertheless, the possibility that hTEE monitoring might have contributed to the difference in survival cannot be excluded. Specifically, hTEE monitoring can allow better visualization of the heart and its function, and perhaps earlier identification of either LVAD or cardiac dysfunction that could possibly be corrected in advance with an appropriate intervention. Future studies with a well-designed control group would be able to better compare and evaluate the effects of routine hTEE

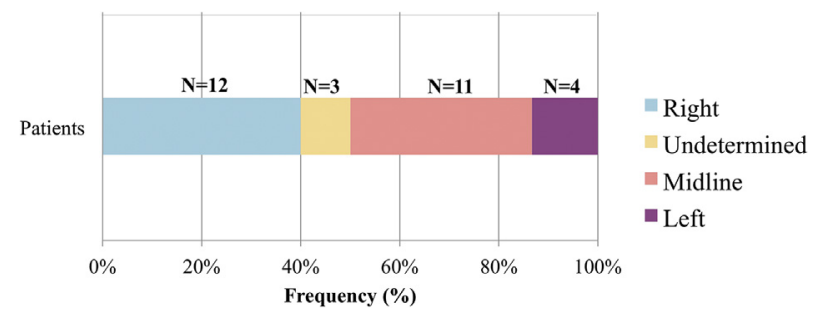

FIGURE 5. Distribution of frequencies of various interventricular septal configurations. 
monitoring on survival, overall costs, and other important outcomes.

The clinical management of patients with an LVAD in the ICU frequently relies on SGC parameters, vital signs, and LVAD settings. Although these parameters are useful for estimating hemodynamic status, they might not provide sufficiently accurate identification of right ventricular failure, ventricular volume status, and interventricular septal position. ${ }^{13}$ Cardiac monitoring, especially of right-sided heart function, is crucial for optimal interventions at the ICU. ${ }^{14}$ Haglund and colleagues ${ }^{7}$ showed that with hTEE clinical management at the ICU was changed in $72 \%$ of studies. The authors emphasized the importance of hTEE monitoring in hemodynamically unstable patients. ${ }^{7}$ Our study shows that hTEE monitoring helps evaluate cardiac function and volume status in the early postoperative period and could lead to changes in clinical management, even in patients who have SGC parameters within normal limits and who initially appear stable based on those parameters. Even though SGC parameters may be normal, indicating hemodynamic stability, hTEE can be abnormal, suggesting the need for intervention. One explanation for this situation could be the influence of the LVAD itself on the measurements and calculations obtained from SGC. Furthermore, the speed of the LVAD can be appropriately adjusted in the ICU using hTEE to improve interventricular septal configuration and ventricular filling.

Limitations of our study are those associated with any retrospective analysis with a small sample size. As noted above, only $7 \%$ of patients received all the necessary interventions based on hTEE findings, which could have affected the SGC parameters recorded at the next time point. Future prospective randomized control studies are needed to eliminate the retrospective bias and allow us to validate the importance of hTEE monitoring in addition to SGC monitoring after LVAD placement and its effects on survival, even in patients with apparent hemodynamic stability.

\section{CONCLUSIONS}

Conventional monitoring in the ICU with SGC may not provide an accurate representation of cardiac function, ventricular volume status, or interventricular septal position in patients with LVAD. Monitoring with hTEE, in addition to SGC, provides improved guidance toward optimal intervention in the ICU setting and should be considered in select patients.

\section{Conflict of Interest Statement}

Authors have nothing to disclose with regard to commercial support.

\section{References}

1. Ranucci M. Which cardiac surgical patients can benefit from placement of a pulmonary artery catheter? Crit Care. 2006;3(10 Suppl):S6.

2. Connors AF Jr, Speroff T, Dawson NV, Thomas C, Harrell FE Jr, Wagner D, et al; SUPPORT investigators. The effectiveness of right heart catheterization in the initial care of critically ill patients. JAMA. 1996;276:889-97.

3. Richard C, Warszawski J, Anguel N, Deye N, Combes A, Barnoud D, et al. Early use of the pulmonary artery catheter and outcomes in patients with shock and acute respiratory distress syndrome: a randomized controlled trial. JAMA. 2003;290:2713-20.

4. Sandham JD, Hull RD, Brant RF, Knox L, Pineo GF, Doig CJ, et al. A randomized, controlled trial of the use of pulmonary-artery catheters in high-risk surgical patients. N Engl J Med. 2003;348:5-14

5. Yu DT, Platt R, Lanken PN, Black E, Sands KE, Schwartz JS, et al. Relationship of pulmonary artery catheter use to mortality and resource utilization in patients with severe sepsis. Crit Care Med. 2003;31:2734-41.

6. Wagner CE, Bick JS, Webster BH, Selby JH, Byrne JG. Use of a miniaturized transesophageal echocardiographic probe in the intensive care unit for diagnosis and treatment of a hemodynamically unstable patient after aortic valve replacement. J Cardiothorac Vasc Anesth. 2012;26:95-7.

7. Haglund NA, Maltais S, Bick JS, Costello W, Keebler M, Davis ME, et al. Hemodynamic transesophageal echocardiography after left ventricular assist device implantation. J Cardiothorac Vasc Anesth. 2014;28:1184-90.

8. Maltais S, Costello WT, Billings FT IV, Bick JS, Byrne JG, Ahmad RM, et al. Episodic monoplane transesophageal echocardiography impacts postoperative management of the cardiac surgery patient. J Cardiothorac Vasc Anesth. 2013; 27:665-9.

9. Cavarocchi NC, Pitcher HT, Yang Q, Karbowski P, Miessau J, Hastings HM, et al Weaning of extracorporeal membrane oxygenation using continuous hemodynamic transesophageal echocardiography. J Thorac Cardiovasc Surg. 2013;146:1474-9.

10. Hirose H, Gupta S, Pitcher H, Miessau J, Yang Q, Yang J, et al. Feasibility of diagnosis of postcardiotomy tamponade by miniaturized transesophageal echocardiography. J Surg Res. 2014;190:276-9.

11. Birati EY, Rame JE. Left ventricular assist device management and complications. Crit Care Clin. 2014;30:607-27.

12. Scalia GM, McCarthy PM, Savage RM, Smedira NG, Thomas JD. Clinical utility of echocardiography in the management of implantable ventricular assist devices. J Am Soc Echocardiogr. 2000;13:754-63.

13. Kavarana MN, Pessin-Minsley MS, Urtecho J, Catanese KA, Flannery M, $\mathrm{Oz} \mathrm{MC}$, et al. Right ventricular dysfunction and organ failure in left ventricular assist device recipients: a continuing problem. Ann Thorac Surg. 2002;73: 745-50.

14. Jardin F, Vieillard-Baron A. Monitoring of right-sided heart function. Curr Opin Crit Care. 2005;11:271-9.

Key Words: hemodynamic monitoring, left ventricular assist device, Swan-Ganz catheter, trasesophageal echocardiography 